\title{
Ascorbic acid: a useful reductant to avoid oxidation of catecholamines in electrophysiological experiments in vitro?
}

\author{
B. Sutor and G. ten Bruggencate \\ Physiologisches Institut der Universität München, Munich (F.R.G.)
}

(Received 5 March 1990; Accepted 25 April 1990)

$\begin{array}{ll}\text { Key words: } & \text { Ascorbic acid; Neocortex; Slice; Intracellular recording; HPLC; Dopamine; Sodium metabi- } \\ \text { sulphate }\end{array}$

The actions of the reductant ascorbic acid on rat neocortical neurons in vitro was investigated by means of intracellular recordings. At a concentration $(500 \mu \mathrm{M})$, which reduced the magnitude of dopamine degradation in oxygen-saturated saline solutions by about $50 \%$, ascorbic acid reversibly depressed synaptic potentials and enhanced direct excitability of cortical neurons. The latter effect was not reversible within the observation period. Ascorbic acid did not alter membrane potential and input resistance of the neurons. On the basis of our results we conclude that ascorbic acid is not a useful reductant to avoid oxidation of catecholamines in oxygen-saturated solutions used in electrophysiological experiments in vitro.

In oxygen-saturated physiological saline solutions, as used in electrophysiological experiments on brain slices, catecholamines are readily oxidized. It has been demonstrated that in these solutions, the noradrenaline concentration declines by $50-60 \%$ within $10 \mathrm{~min}$ [2]. Such a pronounced decrease in the catecholamine content of the superfusing medium results in overestimations of effective catecholamine concentrations. Furthermore, the oxidation of catecholamines yields metabolites which might be biologically active. Therefore, in order to avoid catecholamine oxidation, it is necessary to include protecting reductants into the bathing solution. In the course of an electrophysiological investigation on the actions of dopamine on rat neocortical neurons in vitro, we chose ascorbic acid (ASC) to protect the amine from oxidation [2]. Preliminary experiments, however, revealed marked effects of ASC on electrophysiological properties of the cells. In addition, using high performance liquid chromatography (HPLC) we found that ASC, even at high concentrations, does not provide a complete protection of DA from oxidation.

Correspondence: B. Sutor, Physiologisches Institut der Universität München, Pettenkofferstrasse 12 , D-8000 Munich 2, F.R.G. 
Coronal slices $(500 \mu \mathrm{m})$ of the rat frontal cortex were prepared as described previously [6]. In the recording chamber, the slices were kept submerged in artificial cerebrospinal fluid (ACSF) consisting of (in mM): $\mathrm{NaCl} 118, \mathrm{KCl} 3, \mathrm{NaH}_{2} \mathrm{PO}_{4} 1.2, \mathrm{CaCl}_{2}$ 2.0, $\mathrm{MgCl}_{2}$ 1.3, $\mathrm{NaHCO}_{3} 25$ and $\mathrm{D}$-glucose 10. The solution was saturated with carbogen $\left(95 \% \mathrm{O}_{2}\right.$ and $\left.5 \% \mathrm{CO}_{2}\right)$ in order to obtain a $\mathrm{pH}$ value of $7.35-7.4$ at a temperature of $32^{\circ} \mathrm{C}$. Intracellular recordings from neurons located in cortical layers II and III were performed by means of glass microelectrodes filled with $3 \mathrm{M} \mathrm{KCl}$ (resistance 50-90 M $\Omega$ ). The recorded signals were amplified using an npi SEC $1 \mathrm{~L}$ amplifier and digitized on-line by means of a laboratory computer system (CED 1401 in conjunction with a Tandon PC/AT 40). The membrane potential and input resistance (determined by hyperpolarizing current pulses, $300 \mathrm{~ms}, 0.2-0.4 \mathrm{nA}$ ) were continuously monitored on a chart recorder. For elicitation of postsynaptic potentials, a bipolar concentric stimulation electrode was positioned in an area of the slice corresponding to cortical layer IV. ASC $(500 \mu \mathrm{M})$ and sodium metabisulphite (Na-MBS, $50 \mu \mathrm{M})$ were applied by addition to the bathing solution.

Isocratic reversed phase ion pair HPLC of DA was performed on an Ultrasphere ODS column $(5 \mu \mathrm{m}, 250 \times 4.6 \mathrm{~mm})$ at room temperature. The eluent consisted of $0.025 \mathrm{M}$ citric acid, $0.025 \mathrm{M} \mathrm{Na} \mathrm{HPO}_{4}, 0.005 \mathrm{M}$ ethylenediaminetetraacetic acid (EDTA, disodium salt), $0.7 \mathrm{mM}$ sodium octanesulphonic acid, and $14 \%$ methanol, $\mathrm{pH}$ 3.4. Only HPLC grade reagents and water were used. The flow rate was adjusted to $1 \mathrm{ml} / \mathrm{min}$ and $\mathrm{DA}$ was measured using an amperometric electrochemical detector (Metrohm, Herisau, Switzerland) at an electrode potential of $0.7 \mathrm{~V}$.

Values are given as mean \pm standard deviation (S.D.). Statistical comparisons of mean values were performed using the paired, two-tailed Student's $t$-test.

In a first series of experiments, the ASC concentration necessary to prevent or reduce oxidation of DA in oxygenized ACSF at $32^{\circ} \mathrm{C}$ was determined using HPLC. A stock solution of DA (dissolved in water) was freshly prepared and added to prewarmed and oxygen-saturated ACSF ( $100 \mathrm{ml})$ in order to give a final concentration of $10 \mu \mathrm{M}$. Aliquots of $500 \mu \mathrm{l}$ of this solution were taken at various times after addition of the amine $(0,10$ and $20 \mathrm{~min})$, added to $500 \mu \mathrm{l}$ of $0.4 \mathrm{mM} \mathrm{Na}-\mathrm{MBS}$ and kept on ice until analysis of the samples by HPLC. These experiments were repeated using ACSF containing various concentrations of ASC. In Fig. 1A, chromatograms obtained from measurements in the presence of ASC $(500 \mu \mathrm{M})$ are shown. The diagram in Fig. 1B summarizes the results from these experiments. Following incubation in an ASC-free ACSF for $20 \mathrm{~min}$, the DA concentration was found to be reduced by $38.1 \pm 0.3 \%(n=3)$. In the presence of ASC $(500 \mu \mathrm{M})$, the dopamine concentration decreased by $23.5 \pm 0.9 \%(n=3)$, indicating that ASC at a concentration of 500 $\mu \mathrm{M}$ reduced the magnitude of DA oxidation by about $50 \%$, but did not provide a complete protection of the catecholamine (see also ref. 5).

In electrophysiological experiments, the effects of ASC $(500 \mu \mathrm{M})$ on neocortical neurons in vitro were investigated. The study is based on data obtained from 12 intracellularly recorded neurons. These cells had a mean resting membrane potential (RMP) of $-82.8 \pm 2.4 \mathrm{mV}$, a mean input resistance $\left(R_{\mathrm{N}}\right)$ of $31.6 \pm 9.5 \mathrm{M} \Omega$, and a mean action potential overshoot of $30.5 \pm 4.4 \mathrm{mV}$. The most obvious ASC-induced 
A ACSF/ASC

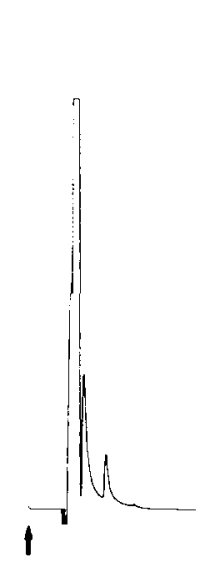

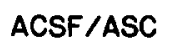

+ DA

$100 \mathrm{pmol}$

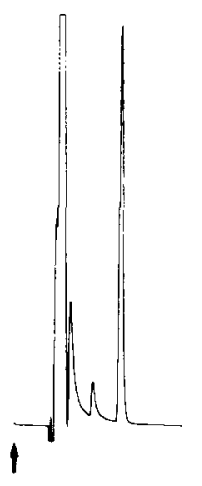

ACSF/ASC

$+D A$

Omin

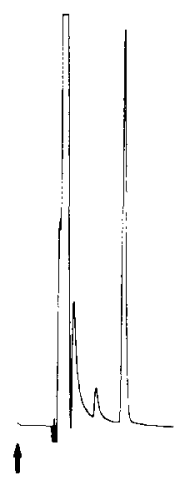

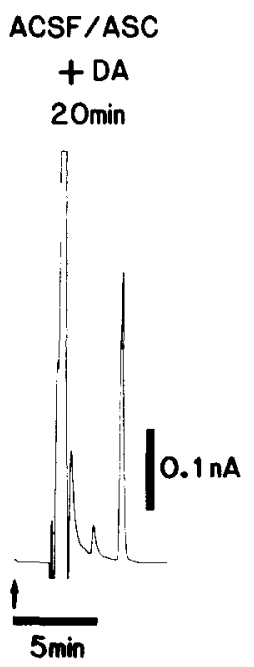

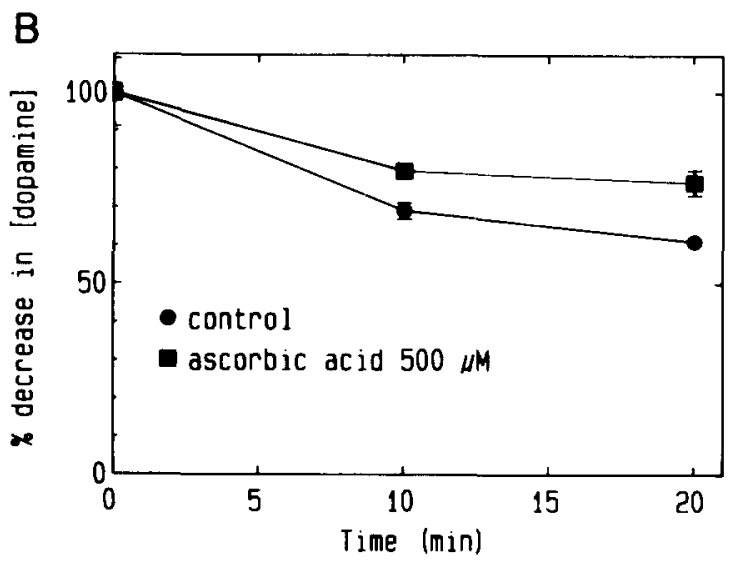

Fig. 1. HPLC analysis of DA degradation in the absence and presence of ASC (500 $\mu \mathrm{M})$. A: chromatograms showing, from the left to the right, the elution of ACSF containing $500 \mu \mathrm{M} \mathrm{ASC}$, the elution of ACSF/ASC containing a 100 pmol DA standard (this amount should be present in the samples at 0 min incubation, see text; the standard was added to ice-cold ACSF/ASC and immediately analysed), the elution of DA incubated in warm and oxygenized ACSF (containing ASC) for 0 min (i.e. the sample was taken immediately after addition of DA to the $\mathrm{ACSF}$ ), and the elution of DA incubated in oxygenized ACSF/ASC for $20 \mathrm{~min}$ at $32^{\circ} \mathrm{C}$. For analysis, $20 \mu \mathrm{l}$ of each sample was applied to the column. DA eluted with a retention time of $6.35 \mathrm{~min}$. Time of injection is indicated by arrows. Each measurement was performed in triplicate. B: plot of the relative decrease in DA concentration as a function of the incubation time. The area under the DA peaks was measured and normalized with respect to the area of the standard peak. Where data points are lacking error bars, the standard deviation was smaller than the size of the symbol.

effect was a partially reversible depression of stimulus-evoked postsynaptic potentials. Fig. 2A depicts the synaptic response of a cortical neuron elicited by electrical stimulation using a subthreshold intensity. Under control conditions, the stimulus 


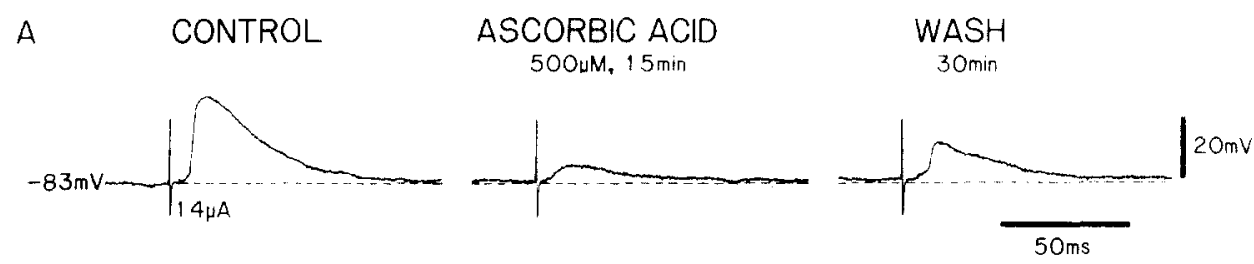

B

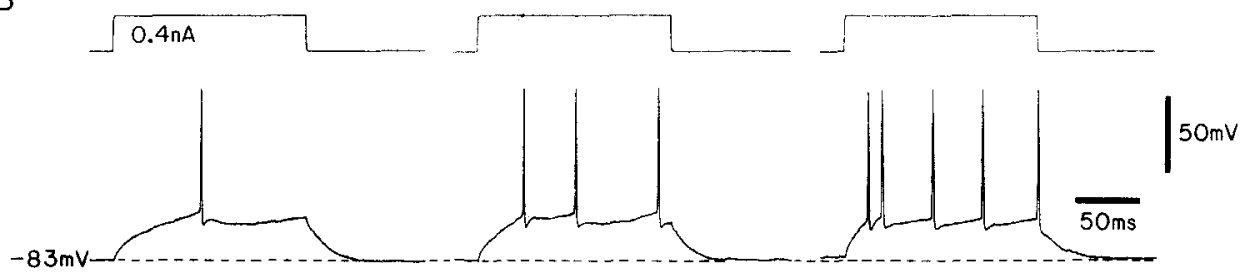

Fig. 2. Effects of ASC on synaptic potentials and direct evoked spike discharge. Intracellular recordings from a neocortical neuron (RMP: $-83 \mathrm{mV}$ ). A: a depolarizing synaptic potential was elicited by subthreshold electrical stimulation (14 $\mu \mathrm{A}$, control). ASC $(500 \mu \mathrm{M})$, applied for $15 \mathrm{~min}$, depressed the amplitude of the synaptic response. This effect was partially reversible upon washout of ASC for $30 \mathrm{~min}$ (wash). $\mathrm{B}$ : recordings from the same cell as in A showing the response of the neuron to a $0.4 \mathrm{nA}$ depolarizing current pulse (150 ms) before (control), during (ASC, $500 \mu \mathrm{M}, 15 \mathrm{~min}$ ), and after (wash) the application of ASC. The dashed lines in A and B indicate the RMP.

produced a large depolarizing postsynaptic potential (Fig. 2A, control), the amplitude of which was found to be reduced by $78 \%$ following the application of ASC at a concentration of $500 \mu \mathrm{M}$ for $15 \mathrm{~min}$ (Fig. 2A, ascorbic acid). Upon washout of ASC for $30 \mathrm{~min}$, a partial recovery of the subthreshold synaptic response was observed (Fig. 2A, wash). Similar observations were made in 6 out of 7 neurons tested.

The second prominent effect of ASC was an enhancement in the neurons' direct excitability. Fig. $2 \mathrm{~B}$ shows the response of a neuron to depolarizing current pulses in the absence and presence of ASC. The amplitude of the current pulse $(0.4 \mathrm{nA}, 150$ $\mathrm{ms}$ ) was adjusted to evoke one action potential (Fig. 2B, control). Following application of ASC ( $500 \mu \mathrm{M}, 15 \mathrm{~min})$, the same pulse evoked 3 action potentials. In contrast to the at least partial recovery of its actions on synaptic potentials, this effect of ASC did not reverse within the observation period $(30 \mathrm{~min}$ wash). On the contrary, a further increase in discharge frequency was observed (Fig. 2B, wash). Similar observations were made following the injection of current pulses which produced a train of action potentials. In 5 neurons, the discharge frequency was determined from the 2nd and 5th interspike interval (ISI) in the absence and presence of ASC. Under normal conditions, the frequencies calculated from the 2nd and 5th ISI were found to be $107.9 \pm 92.1 \mathrm{~Hz}$ and $25.9 \pm 11.2 \mathrm{~Hz}$, respectively, indicating frequency adaptation. In the presence of ASC $(500 \mu \mathrm{M})$, significant alterations in these values were observed (2nd ISI: $127 \pm 89.4 \mathrm{~Hz}$ (significant at $P<0.05$ ); 5th ISI: $34.4 \pm 12.9$ $\mathrm{Hz}$ (significant at $P<0.001$ )). The possibility that the non-reversibility of the 

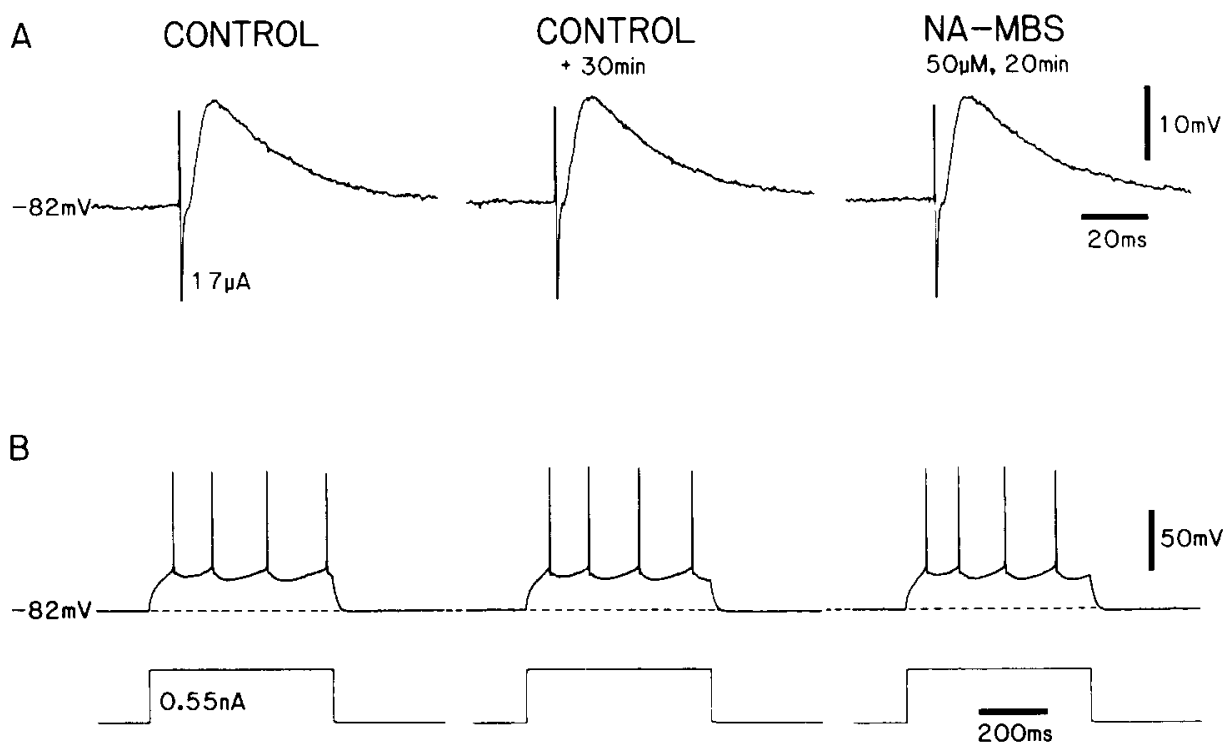

Fig. 3. Effects of Na-MBS on synaptic potentials and direct evoke spike discharge. Intracellular recording from a neocortical neuron (RMP: $-82 \mathrm{mV}$ ). A: A synaptic potential was induced by stimulation using a subthreshold intensity (17 $\mu \mathrm{A}$, control). Following an additional control period of $30 \mathrm{~min}$ (control +30 min), no change in the amplitude of the synaptic response was observed. Upon application of Na-MBS $(50 \mu \mathrm{M})$ for $20 \mathrm{~min}$, the amplitude of the synaptic potential remained unaltered. B: recordings from the same cell. Spike discharge was evoked by injection of a depolarizing current pulse $(0.55 \mathrm{nA}, 500 \mathrm{~ms})$. Two control recordings, taken at an interval of $30 \mathrm{~min}$, are shown (control and control $+30 \mathrm{~min}$, respectively) Following the application of Na-MBS $(50 \mu \mathrm{M}, 20 \mathrm{~min})$, no change in the discharge occurred. The dashed line indicates the RMP.

enhanced direct excitability was due to an ASC-independent progressive increase in action potential frequency was excluded by recordings from cells where Na-MBS was applied instead of ASC. In these neurons, no time-dependent change in the action potential frequency was observed (see Fig. 3B).

While reducing synaptic potentials and enhancing direct excitability, ASC (500 $\mu \mathrm{M})$ did not significantly affect the neurons' membrane potentials (control: $-82.3 \pm 2.7 \mathrm{mV}$; ASC: $-81.4 \pm 3.0 \mathrm{mV} ; n=10$ ). Similarly, ASC had no significant effects on the $R_{\mathrm{N}}$ (control: $30.5 \pm 8.6 \mathrm{M} \Omega$; ASC: $30.2 \pm 8.8 \mathrm{M} \Omega ; n=10$ ). This also argues against slow, ASC-independent changes of neuronal properties.

Since Na-MBS is commonly used as a protecting reductant in HPLC analysis of catecholamines (see ref. 3), we investigated the effects of this substance on the electrophysiological properties of neocortical neurons in vitro. In all cells tested $(n=4), \mathrm{Na}-$ MBS $(50 \mu \mathrm{M})$ neither influenced postsynaptic potentials (Fig. 3A) nor current-pulse evoked spike discharge (Fig. 3B) (see also ref. 1). In addition, no effect on membrane potential and $R_{\mathrm{N}}$ was observed. In the presence of Na-MBS $(50 \mu \mathrm{M})$, the DA concentration declines by $20.6 \pm 1.6 \%(n=3)$, as revealed by HPLC analysis. At higher $\mathrm{Na}$ MBS concentrations ( $200-400 \mu \mathrm{M})$, a complete protection of DA from oxidation was 
observed. However, at these concentrations, Na-MBS affected neuronal properties similar to ASC (data not shown).

These experiments demonstrated pronounced effects of ASC on rat neocortical neurons in vitro. At a concentration $(500 \mu \mathrm{M})$ which reduced the magnitude of DA oxidation in ACSF by about $50 \%$, depression of synaptic potentials and enhancement of direct excitability was observed. Therefore, ASC seems to be not useful as a reductant to protect catecholamines from oxidation in ACSF used in electrophysiological experiments. The mechanism of action of ASC is unclear. It is known that ASC is a cosubstrate of hydroxylation reactions in a number of metabolic pathways. Furthermore, ASC can act as an artificial electron acceptor, thereby influencing oxidative phosphorylation and the production of ATP. In addition, it promotes the generation of free radicals (see ref. 4). An alternative to ASC is Na-MBS, although this substance does not provide a significantly better protection of DA. However, at a concentration which was equipotent to $500 \mu \mathrm{M}$ ASC in preventing DA from oxidation, Na-MBS did not alter electrophysiological properties of neocortical neurons.

This work was supported by the Deutsche Forschungsgemeinschaft ( $\mathrm{Su} \mathrm{104/1-1).}$

1 Akaike, A., Ohno, Y., Sasa, M. and Takaori, S., Excitatory and inhibitory effects of dopamine on neuronal activity of the caudate nucleus in vitro, Brain Res., 418 (1987) 262-272.

2 Hughes, I.E. and Smith, J.A., The stability of noradrenaline in physiological saline solution, J. Pharm. Pharmacol., 30 (1978) 124-125.

3 Marsden, C.A. and Joseph, M.H., Biogenic amines. In C.K. Lim (Ed.), HPLC of Small Molecules, IRL Press, Oxford, 1986, pp. 29-47.

4 Metzler, D.E., Biochemistry. The Chemical Reactions of Living Cells, Academic Press, New York, 1977 , pp. 582-628.

5 Penit-Soria, J., Audinat, E. and Crepel, F., Excitation of rat prefrontal cortical neurons by dopamine: an in vitro electrophysiological study, Brain Res., 425 (1987) 263-274.

6 Sutor, B. and Hablitz, J.J., EPSPs in rat neocortical neurons in vitro. I. Electrophysiological evidence for two distinct EPSPs, J. Neurophysiol., 61 (1989) 607-620. 